\title{
The path to surgery in carcinoid heart disease: a retrospective study and a multidisciplinary proposal of a new algorithm
}

Philippe Mortelmans $\mathrm{MD}^{\mathrm{a}^{*}}$, Marie-Christine Herregods MD PhD ${ }^{\mathrm{b}}$, Filip Rega $\mathrm{MD} \mathrm{PhD}^{\mathrm{c}}$ and Philippe Timmermans $\mathrm{MD}^{\mathrm{d}}$

a Cardiology Department, University Hospitals Leuven, Herestraat 49 Leuven, Belgium, philippe.mortelmans@uzleuven.be

${ }^{\mathrm{b}}$ Cardiology Department, University Hospitals Leuven, Herestraat 49 Leuven, Belgium, marie-christine.herregods@uzleuven.be

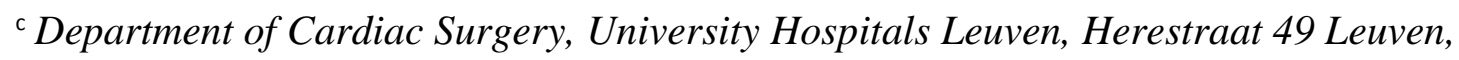
Belgium, filip.rega@uzleuven.be

${ }^{d}$ Cardiology Department, Jessa Hospital Hasselt, Stadsomvaart 11, Hasselt, Belgium, philippe.jr.timmermans@jessazh.be

*corresponding author 


\section{Background}

Development of Carcinoid Heart Disease (CHD) is the major negative prognostic factor in patients with the carcinoid syndrome. The only effective treatment is valve replacement. However, the selection of candidates and determination of optimal timing remain unclear. Considerable variability in local screening and treatment strategies exist.

\section{Methods}

In this single center study we retrospectively analyzed the diagnostic process and outcome of all CHD patients who underwent valve surgery between 2000 and 2016. We propose a new CHD screening and management algorithm.

\section{Results}

All patients $(n=15)$, mean age $64 \pm 7$, underwent tricuspid valve surgery. In 14 of them (93\%) an additional valve was replaced. In only a minority of patients (27\%) CHD diagnosis was established by screening. Survival after 1, 3, 12 and 24 months was 93\%, 80\%, 53\% and 33\% respectively. Causes of death included infections and critical illness immediately postoperatively, and tumor progression and right heart failure in the longer term. There was a trend $(\mathrm{p}=0.099)$ towards better preoperative right ventricular function in the patients who survived more than 12 months postoperatively (TAPSE $20 \mathrm{~mm} \pm 4$ ) compared to those who died between 3 to 12 months after surgery (TAPSE $16 \mathrm{~mm} \pm 1$ ). The former group had a shorter mean interval from diagnosis of the carcinoid syndrome to cardiac diagnosis than the latter (13 versus 105 months, $\mathrm{p}=0.014$ ).

\section{Conclusion}

Mortality after valve replacement for CHD remains high. A probably underestimated cause is late referral for cardiac surgery. We propose a systematic, multidisciplinary approach to all carcinoid syndrome patients.

Key words: Carcinoid heart disease; carcinoid syndrome; valve replacement; Neuroendocrine Tumor 


\section{Introduction}

Neuroendocrine tumors (NET) are epithelial neoplasms with predominant neuroendocrine differentiation, arising in the gastrointestinal tract or elsewhere. By secreting serotonin and other vasoactive substances they can cause the rare carcinoid syndrome (0.5 -2.0 cases / million / year), characterized by diarrhea and flushing [1]. Carcinoid heart disease (CHD) can arise in case of a liver metabolism bypass, most frequently hepatic metastasis. Other possibilities include extensive retroperitoneal lymph node invasion, ovarian involvement or primary bronchial disease [2].

CHD mainly affects the right-sided heart valves. Paraneoplastic fibrosis causes retraction, reduced mobility and eventually fixation of the leaflets in a semi-open position, leading to a combination of regurgitation and stenosis. In a later stadium, right heart failure can develop [3]. In the absence of a significant right-to-left shunt the left heart valves are mostly spared thanks to the filter effect of the lung circulation [2].

Development of CHD with valve involvement is the major negative prognostic factor in patients with the carcinoid syndrome [4]. The only effective cardiac treatment is valve replacement, contributing to better symptom control and survival $[5,6]$. However, the selection of candidates and determination of optimal timing remain unclear. Presymptomatic valve operation has not been associated with late survival benefit in one study [7]. Prospective trials are not available for this rare entity and considerable variability in local screening, diagnosing, treatment and follow-up strategies exist [8].

A recently published expert statement recommends a NT-proBNP based algorithm for follow-up of NET patients and screening for carcinoid heart disease [8]. As these tests are not reimbursed in Belgium, it is not applicable in clinical practice. 
We examined the diagnostic process and outcome of CHD patients who underwent valve surgery, in order to define limitations in current practice. Based on these findings we developed a feasible, multidisciplinary supported screening and treatment algorithm for CHD patients adapted to the Belgian context.

\section{Methods}

We retrospectively analyzed the hospital electronic medical record system for all CHD patients who underwent valve replacement in the largest tertiary cardiology and oncology center in Belgium between 2000 and 2016. Last follow-up was obtained in October 2017. Special attention was paid to the diagnostic chronology and the screening examinations in each patient. Once the timing of the cardiac diagnosis had been retrieved, the reports from the months before were retrospectively analyzed to look for clues of heart failure. If elements were missing, the referring hospitals were contacted. A multidisciplinary working group, consisting of cardiac surgeons, NET-specialists and cardiologists with expertise in cardiooncology and valve disease, proposed a new clinical algorithm. For statistical analysis the two-sided Wilcoxon rank-sum test was used. A probability value (p) of $<0.05$ was considered statistically significant. The study was approved by the institution's local ethics committee.

\section{Results}

\section{Baseline characteristics (table 1)}

Fifteen patients were included, with slight male predominance ( 9 men versus 6 women). Four of them had a significant cardiac history, mostly supraventricular arrhythmia, but 1 patient 
had undergone coronary artery bypass surgery. Most NET's originated from the midgut (13 patients $-87 \%$ ), in the others the primary location remained unknown despite extensive medical imaging. Thirteen patients developed liver metastases, the two remaining patients had metastatic ovarian disease or extensive retroperitoneal lymph node invasion. Serum chromogranin levels at the time of NET diagnosis varied from nearly normal $(239 \mu \mathrm{g} / \mathrm{L})$ to extremely elevated (96 $000 \mu \mathrm{g} / \mathrm{L})$. Initial 5-hydroxyindoleacetic acid (5-HIAA), a metabolite of serotonin, in a 24-hour urine collection was slightly or substantially elevated in all patients (minimum 10mg, maximum 265mg, median 96mg, normal $<9.0 \mathrm{mg}$ ).

\section{Timing of NET and CHD diagnosis (table 2 and 3)}

11 out of 15 patients were in follow up because of a NET when CHD developed, whereas 4 patients with an unknown NET, were initialy diagnosed with cardiac involvement. In the former group, the time interval from diagnosis of NET (with carcinoid syndrome) until positive transthoracic echocardiography (TTE) ranged from 259 days (8.6 months) to 6672 days (more than 18 years), with a mean of 1810 days (5 years). Cardiac complaints can retrospectively be suspected approximately 1 to 11 months earlier. Other time intervals between diagnostic or therapeutic acts and clinical events are listed in table 2.

Symptoms and signs of heart failure were present in $87 \%$ of studied cases (13/15) at the time of cardiac diagnosis by TTE. This exam was performed for screening reasons in 4 patients (27\%), while in the remaining part the indication was based on cardiac complaints or clinical clues. In 10 cases we found a negative TTE somewhere in the NET follow-up process, performed a few days till 13 years (4739 days) after onset of the carcinoid syndrome (median 222 days/7.4 months). In this group of patients, 9 received serial TTE's, with screening intervals ranging from 1 to 3 years. In 13 patients (semi-)urgent cardiac surgery was 
scheduled and valve replacement was performed between 0.5 and 13 months after CHD diagnosis. Two patients were followed up and operated on approximately 2 and 3 years later respectively.

\section{Valvular heart disease and surgery (table 4, figure 1)}

At the moment of surgery all patients had severe tricuspid regurgitation (TR). Moderate tricuspid stenosis (TS) was documented in one patient (mean gradient $4.4 \mathrm{mmHg}$ ). The pulmonary valve demonstrated moderate regurgitation in 9 subjects and mild (peak gradient < $36 \mathrm{mmHg}$ ) stenosis in 7 patients. Signs of RV dilatation (based on basal and midventricular diameter exceeding $41 \mathrm{~mm}$ and $35 \mathrm{~mm}$ respectively) and dysfunction (TAPSE [tricuspid annular plane systolic excursion] $<17 \mathrm{~mm})$ were present in $10(67 \%)$ and $5(33 \%)$ patients respectively. More than mild left sided valvular disease was noted in 6 patients, but in only one of them these findings were based on a non-degenerative, i.e. NET related mechanism. It's worth mentioning that this patient had pulmonary but no hepatic metastases.

All patients $(n=15)$, mean age $64 \pm 7$, underwent tricuspid valve surgery. In 14 of them (93\%) an additional valve was replaced, most often the pulmonary valve $(n=12)$. Two patients underwent quadruple valve replacement, one of them for pathologically proven left sided carcinoid involvement. Double valve replacement was combined with coronary artery bypass grafting in 2 cases. Only bioprostheses were used.

\section{Outcome (table 5 and 6)}

Survival after 1, 3, 12 and 24 months was $93 \%, 80 \%, 53 \%$ and $33 \%$ respectively. From those who survived the surgical admission (11 patients), $70 \%$ experienced an improvement of the functional status to NYHA I or II at time of the first follow-up. In the patients who did not 
improve, mortality was $100 \%$ six months after surgery (data not shown).

Perioperative mortality, defined as death within 30 days, was $7 \%$ (1/15). Three patients died in the first three months, almost exclusively due to infectious complications associated with prolonged critical illness $(n=3)$. From the 11 patients who left the hospital, 4 died because of tumor progression and 3 cases were considered cardiac deaths. At the time of data analysis, after a mean follow-up of 5.7 years, two patients were still alive, being 2 and 3.5 years after surgery. Longest postoperative survivals were 6 and 14 years (data not shown).

We supposed a more or less stabilized condition three months after surgery, and we therefore divided our population according to this cut-off. There was a trend $(\mathrm{p}=0.099)$ towards better mean preoperative right ventricular (RV) function in the patients who survived more than 12 months postoperatively (mean TAPSE 20mm \pm 4 ) compared to those who died between 3 to 12 months after surgery (mean TAPSE $16 \mathrm{~mm} \pm 1$ ). The former group had a shorter mean interval from diagnosis of the carcinoid syndrome to cardiac diagnosis than the latter (13 versus 105 months, $\mathrm{p}=0.014$ ). When comparing the preoperative chromogranin levels in the groups defined by death before 3 months $(29887 \mu \mathrm{g} / \mathrm{L})$, between 3 and 12 months $(14453 \mu \mathrm{g} / \mathrm{L})$ and after 12 months $(11930 \mu \mathrm{g} / \mathrm{L})$, a non-significant evolution (worse outcome when higher chromogranin) was observed. Mean postoperative survival tended to be lower in the triple/quadruple valve group (165 days) compared to the single or double intervention group (1145 days, $\mathrm{p}=0.055$ ).

\section{Discussion}




\section{Diagnosis and outcome}

Peri-operative 30 day-mortality was $7 \%$ (1 patient) in our study, which is in line with the most recent numbers (5-18\%) summarized by Dobson et al [6]. Survival after 1 year and 2 years was $53 \%$ and $33 \%$ respectively. These numbers are slightly lower than in other papers, which mention a 1 year survival of $56-71 \%$ and a 2 year survival of $38-44 \%[6,7,9]$. On the other hand, two of our patients survived 6 to 14 years.

Considering the low patient number in this (and other) retrospective studies, the available data clearly reflect the variable course of disease, but also the wide variety in clinical management of these patients.

Another important factor is the relative high percentage of combined operations in our patients compared to other series $[9,10]$. All patients underwent tricuspid valve replacement because of severe regurgitation, compatible with the well-known finding that the tricuspid valve is most frequently affected [11]. In 93\% of our study group at least one additional valve was replaced. Three subjects underwent triple and two patients even quadruple valve surgery. In this triple/quadruple group we noted a trend toward shorter postoperative survival, probably due to a more severe stadium of CHD. Late referral for surgery cannot be excluded as a contributing factor.

In hospital mortality was almost exclusively related to infectious complications and critical illness, underlining the importance of the preoperative general condition of these palliative oncology patients. After discharge, tumor progression and right heart failure were approximately equally important as causes of death, as was reported before [6]. Indeed, tumor progression is expected because a definite cure of the underlying disease is not available. 


\section{Lack of standardization}

To the best of our knowledge this is the first study with the prespecified goal to document the relative timing of events in surgically managed CHD patients, including NET diagnosis, emerging carcinoid syndrome, development of cardiac complaints, establishing the cardiac involvement and ultimately performing surgery.

NETs are known to have a heterogeneous natural history. Our data clearly point out that the time intervals of the classic sequence NET-carcinoid syndrome-CHD-heart failure can vary considerably. This is demonstrated by the interval between development of the carcinoid syndrome and CHD (defined by a positive TTE), ranging in our series from 259 days (8.6 months) till 6672 days (18 years). Interestingly, in one patient the time interval between the last negative and the first positive TTE was only 5 months (data not shown), pointing again to the unpredictable nature of the disease, as well as to the need of expertise in evaluating CHD echo images to avoid missing subtle abnormalities.

CHD was the primary presentation of the NET in 4 of our patients $(27 \%)$, which is comparable to the $20 \%$ mentioned by Bernheim et al [2]. In only a minority of patients (27\%) CHD diagnosis was established by screening, although in most patients with known carcinoid syndrome (10 out of 11), a negative (screening) TTE was found somewhere in the NET follow-up process. Remarkably, this exam was performed a few days till 13 years (4739 days) after onset of the carcinoid syndrome and, if negative, was repeated after an interval ranging from 1 to 3 years. We found that most cases (13/15) already had signs or symptoms suggestive of heart failure, indicating that the pre-clinical stadium had been missed. 
These findings confirm the difficulty of differentiating emerging heart failure from cancer related pathologies (e.g. general fatigue, hypoalbuminemia, hepatic failure, malignant venous obstruction, ...) during routine oncology follow-up, without additional tools. In addition, there seems to be a large variability in screening practices, even in the same institution. The lack of standardization is also reflected in the time interval between CHD diagnosis with surgical indication, and the operation day (1 to 13 months).

Early surgical intervention may lead to a better outcome, given the known predictors of mortality after CHD operation, such as age, NYHA class, need for diuretics, right heart failure and left-sided valvular disease, all of which are likely to progress when postponing surgery $[4,7]$.

The shorter mean interval from onset of the carcinoid syndrome to cardiac diagnosis in patients who survived more than 12 months postoperatively compared to those who died between 3 to 12 months after surgery (13 versus 105 months), can be interpreted in the same context. Although it is unlikely that in the 3 to 12 months - group the cardiac involvement could stay undetected for 105 months, it seems that a significant delay in diagnosis was present, at least in part due to suboptimal screening.

Therefore, timely diagnosis of CHD is vital. We believe that this goal can be achieved by adhering to a prespecified diagnostic flowchart, reducing variability and diagnostic delay in a busy oncology clinic.

\section{Proposal of a new follow-up algorithm for NET and CHD patients (figure 2 and 3)}

We propose a new CHD management algorithm adapted to the Belgian context, where 
biomarkers as NT-proBNP are not reimbursed and patient cost for TTE is 10 times lower. A multidisciplinary approach by a team consisting of cardiologists with NET expertise, cardiac surgeons, oncologists and gastroenterologists is crucial.

Two dimensional TTE remains the cornerstone for serial follow-up and screening in CHD [3]. Although experience with 3D TTE in these patients is limited, this technique can probably add useful information. TTE should be performed in all patients with liver metastasis, the carcinoid syndrome or involvement of ovary, lung or retroperitoneal lymph nodes. Another screening indication should be a urinary 5-hydroxyindoleacetic acid $>300 \mu \mathrm{mol} / 24 \mathrm{~h}$ (ca. $57.4 \mathrm{mg} / 24 \mathrm{~h}$ ), as this breakdown product of serotonin has been shown to be an independent predictor of the development or progression of carcinoid heart disease [12]. When available, an elevated NT-proBNP (cut-off $260 \mathrm{pg} / \mathrm{ml}$ ) warrants TTE [8]. If the echocardiogram is considered negative, it should be repeated at least once a year.

TTE images of possible CHD patients should be evaluated by a cardiologist with specific experience in the field, given the rarity of the disease. In case of unsatisfying image quality, additional exams should be considered: transesophageal echo (leaflet morphology, presence of an intra-cardiac right-to-left shunt), CT (assessment of the right heart chambers and valves) or MRI (reproducible anatomical and functional information). In patients with evolving exercise intolerance but only moderate valvular disease and an apparently normal right ventricle, we propose an exercise MRI, the feasibility of which has been demonstrated [13]. If RV stroke volume does not increase, emerging heart failure due to CHD is confirmed and surgery should be considered (figure 3).

There is no superior TTE score for detecting CHD [14], nor a consensus parameter for evaluation of RV function [15]. We propose systematically describing morphology and 
function of the right ventricle and the right heart valves by using multiple parameters. Evaluating the tricuspid valve in all available echo windows, including the modified parasternal long axis view to visualize the right ventricular inflow view, is mandatory.

Criteria to be determined are listed in table 7, cut-offs for abnormality as suggested by Lang et al [16].

Timing of surgery in CHD patients remains under debate. Accepted indications are symptomatic CHD, RV dilatation or dysfunction and planned hepatic surgery. It should be kept in mind that valve replacement does not control the underlying disease. Major surgery and subsequent revalidation should be withheld from patients with numerous cardiac and noncardiac comorbidities, which jeopardize postoperative prognosis. A minimal non cardiac prognosis of 12 months has been suggested $[2,6,8]$.

\section{Limitations}

Our study is limited by small patient numbers and its retrospective design. We only evaluated the screening protocols used in patients who underwent surgery, which is prone to selection bias. Evaluation of peri- and postoperative management for CHD surgery and their influence on outcome fell out of the scope of our research.

\section{Conclusion}

In conclusion, mortality after valve surgery for CHD remains high. A probably underestimated contributing cause is late cardiac referral of these oncology patients, whose poor general condition makes differentiation between general fatigue and heart failure symptoms challenging without echocardiography. There is considerable variability in cardiac screening and follow-up of patients at risk for CHD development. We propose a systematic, 
multidisciplinary approach to all carcinoid syndrome patients. Our new algorithm may prevent a delay in the diagnosis of CHD, leading to earlier referral for cardiac surgery and hence better outcome. 
[1] Jensen R. Endocrine Tumors of the Gastrointestinal Tract and Pancreas. In: Lango D, Kasper D, Jameson L, et al., editors. Harrison's Princ. Intern. Med. 18th ed. McGraw-Hill; 2012. p. 3057.

[2] Bernheim AM, Connolly HM, Hobday TJ, et al. Carcinoid heart disease. Prog. Cardiovasc. Dis. 2007;49:439-451.

[3] Dobson R, Cuthbertson DJ, Burgess MI. The optimal use of cardiac imaging in the quantification of carcinoid heart disease. Endocr. Relat. Cancer. 2013;20.

[4] Møller JE, Pellikka PA, Bernheim AM, et al. Prognosis of carcinoid heart disease: Analysis of 200 cases over two decades. Circulation. 2005;112:3320-3327.

[5] Connolly HM, Nishimura RA, Smzth HC, et al. Outcome of cardiac surgery for carcinoid heart disease. J. Am. Coll. Cardiol. 1995;25:410-416.

[6] Dobson R, Burgess MI, Pritchard DM, et al. The clinical presentation and management of carcinoid heart disease. Int. J. Cardiol. 2014;173:29-32.

[7] Connolly HM, Schaff H V., Abel MD, et al. Early and late outcomes of surgical treatment in carcinoid heart disease. J. Am. Coll. Cardiol. 2015;66:2189-2196.

[8] Davar J, Connolly HM, Caplin ME, et al. Diagnosing and Managing Carcinoid Heart Disease in Patients With Neuroendocrine Tumors: An Expert Statement. J. Am. Coll. Cardiol. 2017;69:1288-1304.

[9] Mokhles P, van Herwerden LA, de Jong PL, et al. Carcinoid heart disease: outcomes after surgical valve replacement. Eur. J. Cardio-Thoracic Surg. 2012;41:1278-1283.

[10] Bhattacharyya S, Raja SG, Toumpanakis C, et al. Outcomes, risks and complications of cardiac surgery for carcinoid heart disease. Eur. J. Cardio-thoracic Surg. 2011;40:168-172.

[11] Luis SA, Pellikka PA. Carcinoid heart disease: Diagnosis and management. Best Pract. Res. Clin. Endocrinol. Metab. 2016;30:149-158.

[12] Bhattacharyya S, Toumpanakis C, Chilkunda D, et al. Risk factors for the development and progression of carcinoid heart disease. Am. J. Cardiol. 2011;107:1221-1226.

[13] Timmermans P. Exercise cardiac MRI with pulmonary artery catheter monitoring in early manifestation of carcinoid heart disease: a first in men case report. 2017. submitted

[14] Dobson R, Cuthbertson DJ, Jones J, et al. Determination of the optimal echocardiographic scoring system to quantify carcinoid heart disease. Neuroendocrinology. 2014;99:85-93.

[15] Haugaa KH, Bergestuen DS, Sahakyan LG, et al. Evaluation of right ventricular dysfunction by myocardial strain echocardiography in patients with intestinal carcinoid disease. J. Am. Soc. Echocardiogr. 2011;24:644-650.

[16] Lang RM, Badano LP, Mor-avi V, et al. Recommendations for Cardiac Chamber Quantification by Echocardiography in Adults : An Update from the American Society of Echocardiography and the European Association of Cardiovascular Imaging. J. Am. Soc. Echocardiogr. 2015;28:139.e14. 